\title{
Surfactant mesophases mediated by colloidal particles
}

\author{
Masayuki Imai $^{1}$, Yukiko Suganuma ${ }^{1}$, Kaori Nakaya ${ }^{1}$ \\ and Shigeyuki Komura ${ }^{1,2}$ \\ ${ }^{1}$ Department of Physics, Faculty of Science, Ochanomizu University, 2-1-1 Otsuka, Bunkyo, \\ Tokyo 112-0012, Japan \\ ${ }^{2}$ Department of Chemistry, Faculty of Science, Tokyo Metropolitan University, \\ 1-1 Minami-Osawa, Hachioji, Tokyo 192-0397, Japan \\ E-mail: imai@phys.ocha.ac.jp
}

Received 22 December 2004, in final form 4 February 2005

Published 22 July 2005

Online at stacks.iop.org/JPhysCM/17/S2929

\begin{abstract}
We have investigated effects of guest colloidal particles on the mesoscopic structures of a surfactant/water system by means of small angle neutron and $\mathrm{x}$-ray scattering techniques. The addition of a small amount of colloidal particles between lamellar slits reduces the undulational fluctuations of lamellae and brings about a lamellar to micelle transition. On the other hand, on addition of colloidal particles into a disordered prolate micelle phase, the micelles deform to a spherical shape. We will discuss these structure changes in terms of the depletion effects due to the presence of colloidal particles.
\end{abstract}

\section{Introduction}

Soft matter forms a variety of mesoscopic structures as a result of a balancing between entropic interactions and energetic interactions. The balance is easily modified by applying external fields such as shear flow and electric field, and the original mesoscopic structure is destabilized. The addition of a small amount of guest particles to the host mesoscopic structure provides a unique external field for controlling the mesoscopic structure. Thus, on adding guest particles, the dominant interactions that stabilize the host soft material structure are modified by an entropic interaction originating from the excluded volume of the guests, which brings about characteristic structural changes. The most familiar entropic interaction is the depletion interaction [1,2], which is an attractive interaction between host particles caused by the reduction of the free volume of the guests. Another interesting entropic interaction induced by guests is reported for lamellar membrane + guest polymer systems [3-6]. Ligoure et al showed that the addition of polymer chains into the lamellar membranes induces a decrease of the smectic compressibility and finally brings about a lamellar-lamellar phase separation [5, 6]. Thus the effective inter-lamellar interaction mediated by the presence of polymer chains is attractive. 
In this study we investigate the structural change of surfactant mesoscopic structures induced by the addition of guest hard sphere particles [7]. The excluded volume effect of the hard spheres may bring about another kind of entropic interaction compared with the case of polymer chains which have soft nature due to the large internal degrees of freedom. We added spherical colloidal particles between lamellar membrane slits and into the disordered micelles phase and followed the change of the mesoscopic structures by means of small angle X-ray scattering (SAXS) and small angle neutron scattering (SANS) techniques. We will show that addition of a small amount of guest colloidal particles brings about unique structural changes due to the excluded volume of the guest particles.

\section{Experiments}

We investigate ternary mixtures of water, $\mathrm{C}_{12} \mathrm{E}_{5}$, and spherical colloid particles. The nonionic surfactant $\mathrm{C}_{12} \mathrm{E}_{5}$ (purity $>98 \%$ ) was purchased from Nikko Chemicals Inc., and used without further purification. The colloidal particles used in this study were of polystyrene latex having the diameter of $160 \AA$, obtained from Magsphere Inc.

The ternary mixture samples for the scattering measurements were prepared by the following procedure. The surfactant and water mixtures $\left(\mathrm{C}_{12} \mathrm{E}_{5} / \mathrm{D}_{2} \mathrm{O}\right.$ for SANS and $\mathrm{C}_{12} \mathrm{E}_{5} / \mathrm{H}_{2} \mathrm{O}$ for SAXS) were homogenized and then the colloidal particles were added to the surfactant solution $\left(\Phi_{\mathrm{C} 12 \mathrm{E} 5}=0.053, \Phi_{\mathrm{C} 12 \mathrm{E} 5}\right.$ : volume fraction of $\left.\mathrm{C}_{12} \mathrm{E}_{5}\right)$ at room temperature (isotropic micelle phase). We waited several weeks to examine the stability of the observed phases, because in the case of lamellar + guest component systems it sometimes takes an extremely long time to equilibrate the phases. The samples of the lamellar phase without the guests are monophasic and slightly cloudy and on the addition of the colloidal particles, the samples became transparent. The homogenized sample was transferred to a cell for scattering measurements and then annealed at the measurement temperature $57^{\circ} \mathrm{C}$ for the lamellar phase experiment and $30^{\circ} \mathrm{C}$ for the disordered micelle phase experiment.

Details of the scattering experiments are given in the preceding paper [7].

\section{Results and discussion}

\subsection{Phase behaviour of the lamellae membranes + colloid particles system}

First we show the characteristic phase behaviours of the lyotropic lamellar membranes + colloidal particles system. Figure 1(a) shows a series of SANS profiles as a function of $\Phi_{\text {col }}\left(\Phi_{\text {col }}\right.$ : volume fraction of colloidal particles) at constant $\Phi_{\mathrm{C} 12 \mathrm{E} 5}=0.053$. Unfortunately, there is no visible Bragg peak in the SANS profile of the lamellar phase without guests, because of the large amplitude of the membrane fluctuations. Then we evaluate the lamellar repeat distance $d$ from the following relationship from the literature [8]:

$$
d=\frac{t}{\Phi_{\mathrm{C} 12 \mathrm{E} 5}}\left(1-\frac{k_{\mathrm{B}} T}{4 \pi \kappa_{0}} \ln \Phi_{\mathrm{C} 12 \mathrm{E} 5}\right)
$$

where $t$ is the thickness of the membrane and $\kappa_{0}$ is the bare elastic bending modulus of a single membrane. Using $t=30 \AA$ and $\kappa_{0}=1.3 k_{\mathrm{B}} T$, we obtained $d\left(\Phi_{\mathrm{C} 12 \mathrm{E} 5}=0.053\right)=666 \AA$. Thus the size of the guest colloid particle is much smaller than the lamellar period. On addition of colloidal particles, the first lamellar peak begins to appear at very low colloid concentration $\Phi_{\text {col }}=0.01 \%$ and the lamellar period estimated from the peak position is $\sim 630 \AA$, which roughly agrees with the value estimated using equation (1). With increasing $\Phi_{\text {col }}$, the first Bragg peak sharpens and the second harmonic emerges. Thus in the presence of colloidal particles, 
(a)

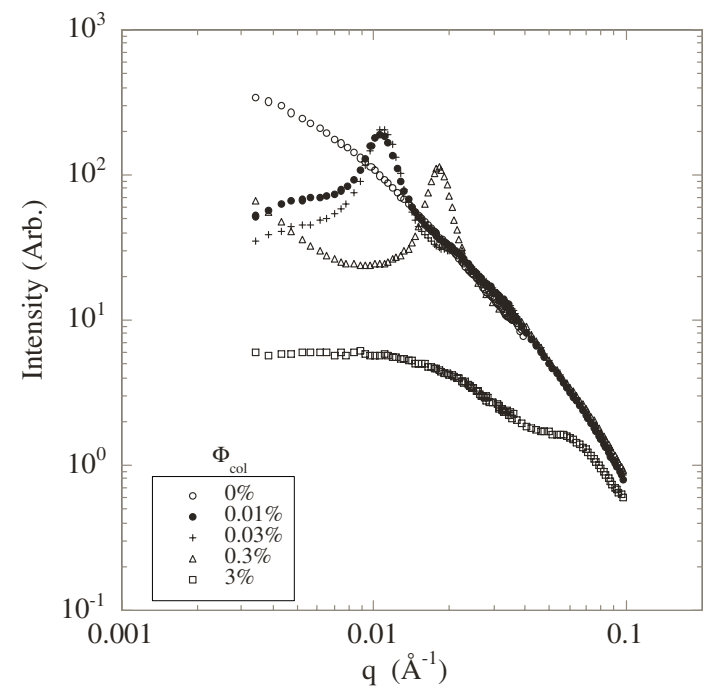

(b)

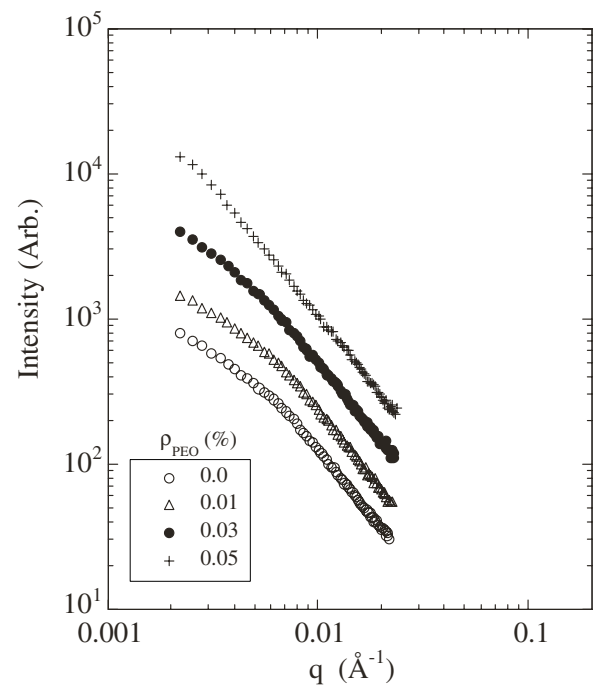

Figure 1. SANS profiles of a lamellar phase confining guest components, colloidal particles (a) and PEO (b), at constant surfactant volume fraction $\Phi_{\mathrm{C} 12 \mathrm{E} 5}=0.053$ as a function of the guest concentration.

the fluctuation of the lamellar membrane is suppressed and the inter-lamellar interactions induced by the addition of the guest colloidal particles are repulsive. Similar modification of inter-lamellar interactions caused by the presence of hard particles has been reported for a lyotropic lamellar with colloidal magnetic particles system [9]. With further increase of $\Phi_{\text {col }}$, the lamellar peaks shift to the higher $q$ side at $\Phi_{\text {col }} \sim 0.3 \%$, which indicates a phase separation to concentrated and swollen lamellae. At $\Phi_{\mathrm{C} 12 \mathrm{E} 5}=3 \%$, the scattering profile shows a drastic change and can be described using the sum of the spherical colloid particle and the spherical micelle form factors. Thus the addition of colloidal particles brings about a lamellar to micelle transition.

It is quite interesting to note that the addition of polymer chains between the lamellar slits brings about an attractive interaction, as revealed by Ligoure et al [4-6]. They found that the addition of the polymer into the smectic bilayer decreases the inter-lamellar repulsive force and finally brings about the lamellar-lamellar phase separation when the layer compression modulus vanishes. We show the change of SANS profiles for $\mathrm{C}_{12} \mathrm{E}_{5}$ lamellar phase upon addition of poly(ethylene oxide) $(\mathrm{PEO})$ at $\Phi_{\mathrm{C} 12 \mathrm{E} 5}=0.053$ in figure 1(b). Upon the addition of PEO chains the scattering functions keep the initial profile until $\rho_{\text {PEO }} \sim 0.03 \%$ ( $\rho_{\text {PEO }}$ : weight fraction of PEO) and then the scattering intensity in the low $q$ region increases with increase of $\rho_{\mathrm{PEO}}$, which is due to the phase separation. The Ligoure model explains the observed phase separation behaviour for the $\mathrm{C}_{12} \mathrm{E}_{5}$ lamellar membrane + polymer system, whereas for the membrane + colloidal particle system the observed strong repulsive force between adjacent lamellar layers and the lamellar-micelle transition cannot be explained by that model. The difference between the addition of polymer and colloid particles is probably attributable to the excluded volume effect of added guest particles; thus the polymer chain has flexible entities and the spherical colloidal particle has rigid entities [7, 9]. In the following we focus on the morphology transition induced by the addition of hard colloidal particles using a simpler system, i.e. the micelle + colloid particle system. 


\subsection{Micelle + colloid particle system}

Here we investigate the effect of guest colloid particles on the morphology of surfactant micelles. It is well known that the shape of disordered surfactant micelles strongly depends on the temperature and concentration of the surfactant solution. In the case of nonionic surfactant/water systems, the micelles have spherical shape at a certain temperature expressed by $T_{\mathrm{c}}-T_{0}$, where $T_{\mathrm{c}}$ is a critical consolution temperature and $T_{0}$ is the characteristic temperature depending on the chemical structure, and above $T_{\mathrm{c}}-T_{0}$ the micelles grow into prolate micelles [10]. We added a small amount of colloidal particles into the disordered prolate micelle phase of the $\mathrm{C}_{12} \mathrm{E}_{5}$ /water system. Figure 2 shows the SAXS profiles as a function of $\Phi_{\text {col }}$. Without colloidal particles the scattering profile is well described using the form factor of a prolate core-shell model expressed by [11]

$$
\begin{aligned}
& \langle P(q)\rangle \equiv \int_{0}^{1}|F(q, x)|^{2} \mathrm{~d} x \\
& F(q, x)=\left(\rho_{\mathrm{c}}-\rho_{\mathrm{m}}\right) \frac{4}{3} \pi a_{\mathrm{i}} R_{\mathrm{i}}^{3}\left[3 \frac{j\left(u_{\mathrm{i}}\right)}{u_{\mathrm{i}}}\right]+\left(\rho_{\mathrm{m}}-\rho_{\mathrm{s}}\right) \frac{4}{3} \pi a_{\mathrm{o}} R_{\mathrm{o}}^{3}\left[3 \frac{j\left(u_{\mathrm{o}}\right)}{u_{\mathrm{o}}}\right] \\
& u_{\mathrm{i}}=q R_{\mathrm{i}} \sqrt{a_{\mathrm{i}}^{2} x^{2}+\left(1-x^{2}\right)}, \quad u_{\mathrm{o}}=q R_{\mathrm{o}} \sqrt{a_{\mathrm{o}}^{2} x^{2}+\left(1-x^{2}\right)}
\end{aligned}
$$

where $R_{\mathrm{o}}$ and $R_{\mathrm{i}}$ are the outer and inner radii of the prolate minor axis, $a_{\mathrm{o}}$ and $a_{\mathrm{i}}$ the ratios of major to minor axes for outer and inner radius, respectively, and $j(x)$ is the first-order spherical Bessel function. The electron densities $\rho_{\mathrm{c}}, \rho_{\mathrm{m}}, \rho_{\mathrm{S}}$ correspond to core, membrane, and solvent, respectively. With increasing $\Phi_{\text {col }}$, the scattering intensity of the lower $q$ side of the form factor peak decreases, indicating that the micelle shape deforms from a prolate to a spherical shape. The result of fitting using equations (2)-(4) is shown in figure 2 by a solid curve for each scattering profile and the $a_{\mathrm{o}}$ and $R_{\mathrm{o}}$ obtained are plotted against $\Phi_{\text {col }}$ in figure 3. With increasing $\Phi_{\text {col }}$ the axis ratio decreases monotonically, keeping $R_{\mathrm{o}}$ constant, and at $\Phi_{\text {col }}=0.01$, the micelle has a nearly spherical shape $\left(a_{0} \sim 1.5\right)$. Thus on addition of colloidal particles, the prolate micelle transforms to a nearly spherical micelle.

We interpret the observed prolate to sphere transition induced by the addition of colloidal particles in terms of the depletion effect $[1,2]$. In this case the system can be described as a mixture of $N_{\mathrm{c}}$ hard sphere colloidal particles with radius $R_{\mathrm{c}}$ and $N_{\mathrm{m}}$ surfactant micelles with the size $\delta\left(R_{\mathrm{c}}>\delta\right)$. The centre of a micelle is excluded from a 'depletion zone' of thickness $\delta$ measured from the surface of a colloidal particle. When the shape of the micelles transforms from prolate to spherical, the size of the micelle (thickness of the depletion zone) decreases and the free volume of the micelles increases. When this free energy gain overcomes the free energy cost of the micelle transformation, the micelles will show the prolate to sphere transition. Thus the total free energy for the micelles + colloidal particles system can be expressed as

$$
F_{\mathrm{m}+\mathrm{c}}=F_{\mathrm{tr}}+n_{\mathrm{s}} f_{\mathrm{mic}}
$$

where $F_{\text {tr }}$ is the translational entropy contribution of a binary mixture of surfactant micelles and colloid particles, $n_{\mathrm{s}}$ is the number of surfactant molecules in a volume $V$, and $f_{\text {mic }}$ is the free energy of a surfactant molecule in a micelle. The mixing free energy is expressed as [11]

$$
F_{\mathrm{tr}}=F_{\mathrm{c}}\left(N_{\mathrm{c}}, V\right)+F_{\mathrm{m}}\left(N_{\mathrm{m}}, \alpha V\right)
$$

where $F_{\mathrm{c}}$ and $F_{\mathrm{m}}$ are the free energies due to the translational entropy of the pure colloid particles system and the pure micelles system, $N_{\mathrm{c}}$ and $N_{\mathrm{m}}$ are the numbers of colloidal particles and micelles in the volume $V$. The free volume fraction $\alpha$ is defined by $V_{\text {free }} \equiv \alpha V$ where 


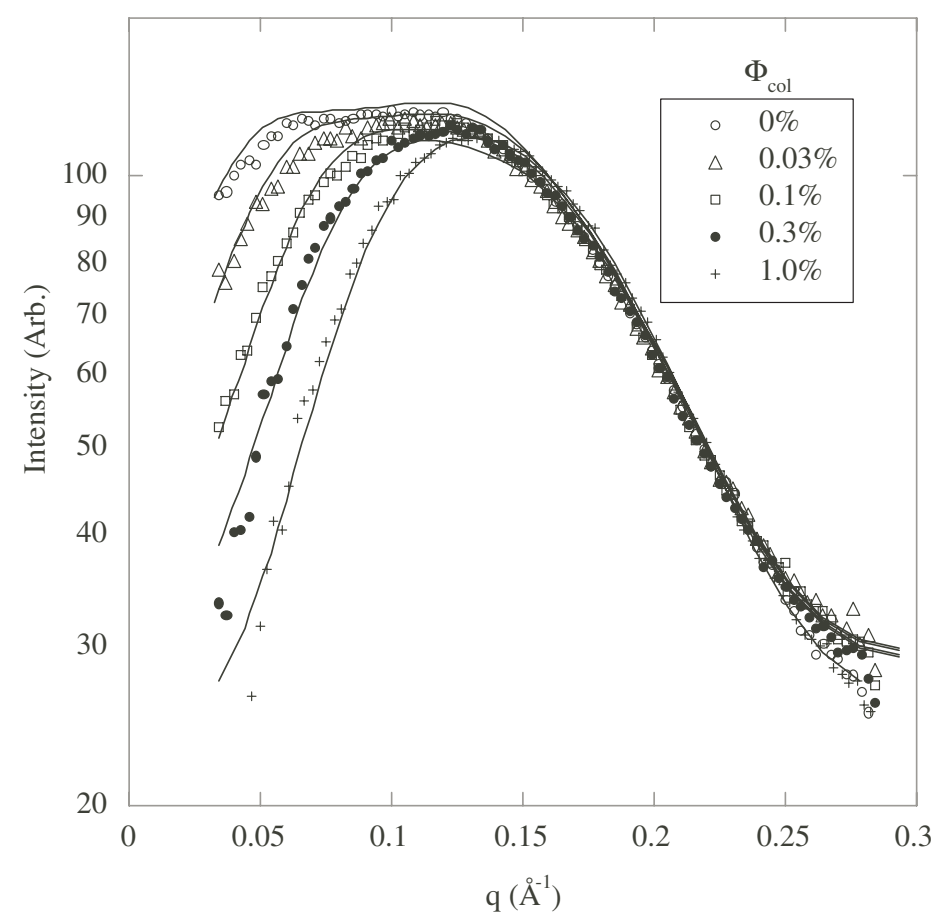

Figure 2. SAXS profiles of the disordered micelle phase at constant surfactant volume fraction $\Phi_{\mathrm{C} 12 \mathrm{E} 5}=0.053$ as a function of the colloidal particle concentration $\Phi_{\text {col }}$.

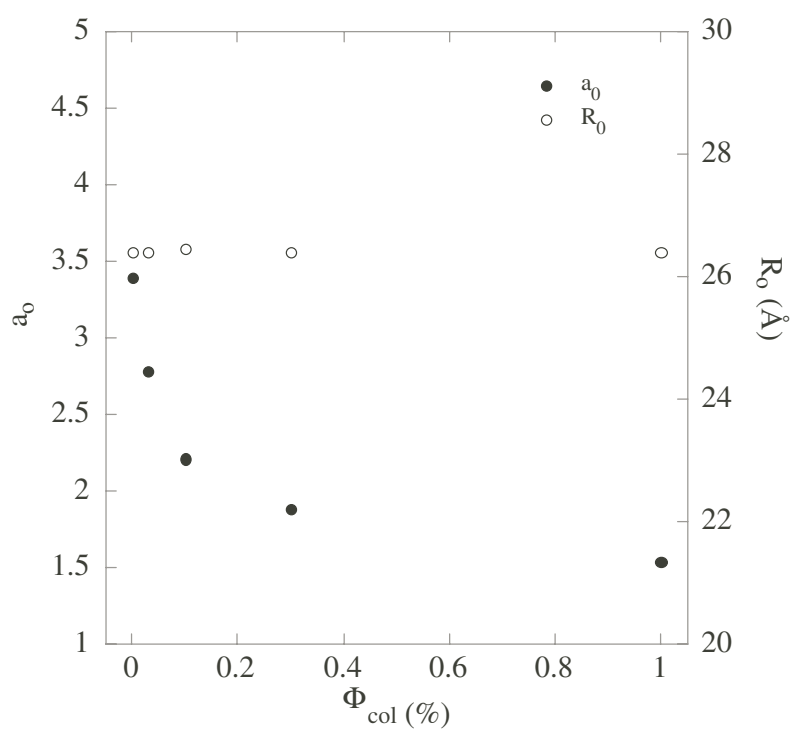

Figure 3. Dependences of the outer radius of the prolate minor axis, $R_{\mathrm{o}}$, and the ratio of major to minor axes for the outer radius, $a_{\mathrm{o}}$, on colloidal particle concentration $\Phi_{\mathrm{col}}$.

$V_{\text {free }}$ is the free volume in which the micelles can move and for simplicity we expressed $V_{\text {free }}$ by

$$
V_{\text {free }}=V-\frac{4}{3} \pi\left(R_{\mathrm{c}}+\delta\right)^{3} N_{\mathrm{c}} .
$$


In order to calculate $V_{\text {free}}$, we approximate the value of $\delta$ using the outer radius of the major axis of the prolate micelle; thus

$$
\delta= \begin{cases}R_{\mathrm{o}} & \text { for spherical micelles } \\ a_{0} R_{\mathrm{o}} & \text { for prolate micelles. }\end{cases}
$$

When we assume that the micelles interact as points with each other, but as hard particles having size $\delta$ with colloidal particles, we can write

$$
F_{\text {mic }}=N_{\mathrm{m}} k_{\mathrm{B}} T \log \left(N_{\mathrm{m}} v_{\mathrm{m}} / V_{\text {free }}\right)
$$

where $v_{\mathrm{m}}$ is the volume of a surfactant micelle. The free energy of hard colloid particles can be expressed by [12]

$$
\begin{aligned}
F_{\mathrm{c}}\left(N_{\mathrm{c}}, V\right) & =\frac{3 k_{\mathrm{B}} T}{4 \pi R_{\mathrm{c}}^{3}} V \Phi_{\mathrm{col}} \int \frac{\left(1+\Phi_{\mathrm{col}}+\Phi_{\mathrm{col}}^{2}\right)}{\Phi_{\mathrm{col}}\left(1-\Phi_{\mathrm{col}}\right)^{3}} \mathrm{~d} \Phi_{\mathrm{col}} \\
& =N_{\mathrm{c}} k_{\mathrm{B}} T\left\{\frac{3}{2\left(1-\Phi_{\mathrm{col}}\right)^{2}}-\log \left(1-\Phi_{\mathrm{col}}\right)+\log \Phi_{\mathrm{col}}\right\} .
\end{aligned}
$$

Unfortunately we do not have an appropriate theoretical free energy model for describing the surfactant micelle; thus we adopt a strong segregation theory of diblock copolymers because the phase behaviour of diblock copolymer is very similar to that of surfactant/water systems. Olmsted and Milner [13] developed a strong segregation theory of micelles based on wedge constituents filled with diblock copolymer. According to their theory, the free energies per molecule for the cylinder and sphere are given by

$$
\begin{aligned}
& f_{\text {cyl }}=f_{0}\left[\frac{2 \varepsilon \phi\left(1-\phi^{1 / 2}\right)^{3}\left(3+\phi^{1 / 2}\right)}{(1-\phi)^{2}}+\frac{2 \phi}{\varepsilon}\right]^{1 / 3} \\
& f_{\text {sph }}=3 f_{0}\left[\frac{\varepsilon \phi^{4 / 3}\left(1-\phi^{1 / 3}\right)^{3}\left(\phi^{2 / 3}+3 \phi^{1 / 3}+6\right)}{10(1-\phi)^{2}}+\frac{\phi}{10 \varepsilon}\right]^{1 / 3} \\
& f_{0}=\left(\frac{27 \pi^{2}}{32}\right)^{1 / 3} \Omega^{1 / 3} \gamma^{2 / 3}\left(\frac{\Omega_{\mathrm{A}} \Omega_{\mathrm{B}}}{R_{\mathrm{A}}^{2} R_{\mathrm{B}}^{2}}\right)^{1 / 6}
\end{aligned}
$$

where $\varepsilon$ is Fredrickson's asymmetry parameter, $\Omega$ is the total volume of the surfactant chain, $\Omega_{\mathrm{A}}$ and $\Omega_{\mathrm{B}}$ are the volumes of hydrophobic chains with end to end distance $R_{\mathrm{A}}$ and hydrophilic chains with end to end distance $R_{\mathrm{B}}$, respectively. The volume fraction of hydrophobic component $\phi$ is expressed by

$$
\phi=\Omega_{\mathrm{A}} / \Omega \text {. }
$$

We approximate the prolate micelle by a spherocylinder model; then the free energy per molecule for the spherocylinder is expressed by

$$
f_{\mathrm{sc}}=\frac{1}{a_{0}} f_{\mathrm{sph}}+\left(1-\frac{1}{a_{0}}\right) f_{\mathrm{cyl}} .
$$

Then we can calculate the free energy of the 'micelles having various axis ratios $a_{0}+$ colloidal particles' mixture system, $F_{\text {mic }+\mathrm{c}}\left(a_{0}, \Phi_{\text {col }}\right)=F_{\mathrm{m}}+n_{\mathrm{s}} f_{\text {mic }}$, as a function of the colloidal particle volume fraction $\Phi_{\mathrm{col}}$. Using the values $\gamma=1.7 \mathrm{mN} \mathrm{m}^{-1}, R_{\mathrm{c}}=80 \AA$, and $R_{\mathrm{o}}=26 \AA$, we plot the normalized free energies, $\left[F_{\text {mic }+\mathrm{c}}\left(a_{0}, \Phi_{\text {col }}\right)-F_{\text {mic }+\mathrm{c}}(1,0)\right] / F_{\text {mic }+\mathrm{c}}(1,0)$, for $a_{0}=1.0,1.5$, $2.0,2.5,3.0$, and 3.5 in figure 4 , where $F_{\text {mic }+c}(1,0)$ is the free energy for spherical micelles without colloidal particles. Here we estimated the molecular volume of the surfactant molecule using literature values [14] and $R_{\mathrm{A}}$ and $R_{\mathrm{B}}$ were assumed to be the surfactant micelle core minor radius and the hydrophilic layer thickness, respectively. In figure 4 we can see a sequential 
(a)

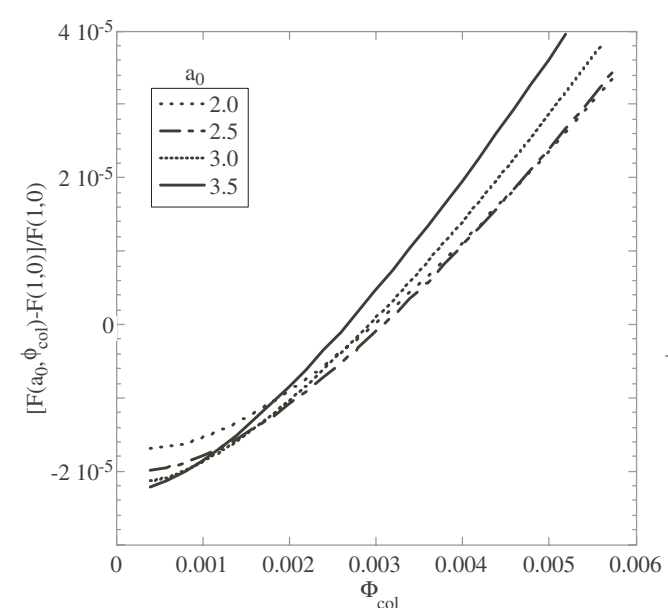

(b)

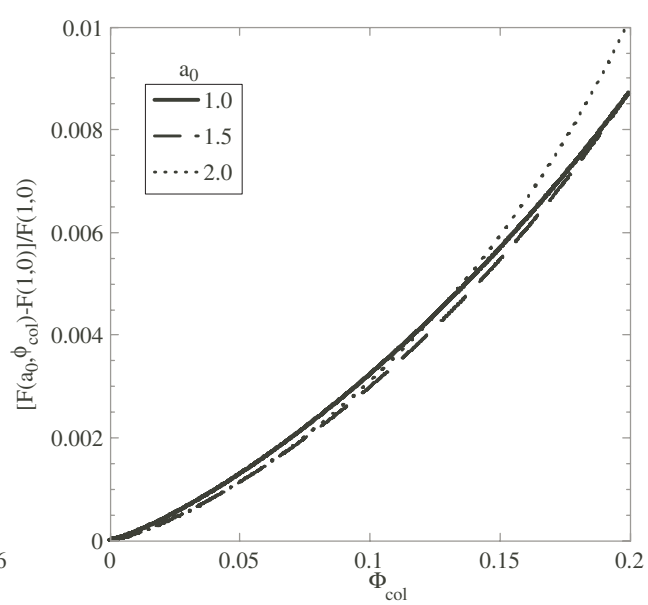

Figure 4. Normalized free energies of a 'micelles having various axis ratios $a_{0}$ ((a) $a_{0}=3.5$, $3.0,2.5,2.0$ and (b) $2.0,1.5,1.0)+$ colloidal particles' mixture system, $\left[F_{\text {mic }+\mathrm{c}}\left(a_{0}, \Phi_{\mathrm{col}}\right)-\right.$ $\left.F_{\text {mic }+\mathrm{c}}(1,0)\right] / F_{\text {mic }+\mathrm{c}}(1,0)$, as a function of the colloidal particle concentration $\Phi_{\text {col }}$.

transition from the prolate micelles $\left(a_{0}=3.5\right)$ to spherical micelles $\left(a_{0}=1.0\right)$ with increasing $\Phi_{\text {col }}$, which agrees with the experimental results. Of course the quantitative behaviour of the theoretical model strongly depends on the parameter values used, especially the ratio of $\delta / R_{\mathrm{c}}$ and the surface tension $\gamma$. In order to analyse the experimental data more quantitatively, we need a refinement of the theoretical model. We will describe this in a forthcoming paper.

\section{References}

[1] Asakura S and Oosawa F 1954 J. Chem. Phys. 221255

[2] Anderson V J and Lekkerkerker H N W 2002 Nature 416811

[3] Daoud M and de Gennes P G 1977 J. Physique 3885

[4] Ligoure C, Bouglet G and Porte G 1993 Phys. Rev. Lett. 713600

[5] Ligoure C, Bouglet G, Porte G and Diat O 1997 J. Physique II 7473

[6] Porcar L, Ligoure C and Marignan J 1997 J. Physique II 7493

[7] Imai M, Mawatari R, Nakaya K and Komura S 2004 Eur. Phys. J. E 13391

[8] Strey R, Schomäcker R, Roux D, Nallet F and Olsson U 1990 J. Chem. Soc. Faraday Trans. 862253

[9] Ponsinet V and Fabre P 1996 J. Physique II 6955

[10] Kato T, Anzi S and Seimiya T 1990 J. Phys. Chem. 947255

[11] Kotlarchyk M and Chen S-H 1983 J. Chem. Phys. 792461

[12] Lekkerkerker H N W and Stroobants A 1993 Physica A 195387

[13] Olmsted P D and Milner S T 1998 Macromolecules 314011

[14] Minewaki K, Kato T, Yoshida H, Imai M and Ito K 2001 Langmuir 171864 